\title{
Photoacoustic and thermoacoustic tomography with both optical and electrical contrasts
}

Geng Ku, Lihong V. Wang

Geng Ku, Lihong V. Wang, "Photoacoustic and thermoacoustic tomography with both optical and electrical contrasts," Proc. SPIE 6086, Photons Plus Ultrasound: Imaging and Sensing 2006: The Seventh Conference on Biomedical Thermoacoustics, Optoacoustics, and Acousto-optics, 608618 (6 March 2006); doi: 10.1117/12.646365 


\title{
Photoacoustic and Thermoacoustic Tomography with both Optical and Electrical Contrasts
}

\author{
Geng $\mathrm{Ku}$ and Lihong V. Wang* \\ Optical Imaging Laboratory, Department of Biomedical Engineering, Texas A\&M University, 3120 \\ TAMU, College Station, TX 77843-3120
}

\begin{abstract}
The absorption of electromagnetic energy causes thermal expansion and induces acoustic waves in biological tissues. Various tissues present particular characteristics in their absorption spectra. To acquire both photoacoustic and thermoacoustic images with multiple contrasts that reflect the absorption of electromagnetic energy, biological tissues are stimulated using laser and microwave pulses, respectively. Muscles with a rich blood supply strongly absorb green optical radiation, which provides excellent optical contrast. High water content tissues, such as connective tissue and muscle tissue, display high contrast to fatty tissues when imaged using microwave radiation. Most cancerous tissues have higher water and ionic concentrations, two characteristics that are also associated with angiogenesis and hemoglobin oxygen saturation. Therefore, cancer diagnosis based on information from tissue properties over an electromagnetic spectrum from microwave to optical bands can be more accurate than was previously available.
\end{abstract}

Keywords: photoacoustic tomography, thermoacoustic tomography, medical imaging, absorption spectroscopy, biological tissue, optical contrast, radiofrequency contrast.

\section{INTRODUCTION}

Photoacoustic tomography (PAT) and thermoacoustic tomography (TAT) are both biological imaging techniques based on the measurement of ultrasonic waves induced by electromagnetic pulses. They can reveal those optical and electrical properties of tissues that are closely related to their physiological and pathological status.(1-4) PAT employs visible or near infrared (NIR) light, while TAT uses electromagnetic radiation in the radiofrequency (RF) or microwave bands. These novel non-invasive and non-ionizing imaging modalities can potentially provide high imaging contrast based on a tissue's rate of absorption of electromagnetic radiation.

Electromagnetic energy is absorbed by biological tissues when an electromagnetic pulse, such as a laser or microwave pulse, is used to irradiate the tissues. Absorption of the electromagnetic energy generates heat and the subsequent expansion causes the emission of acoustic waves, which is called the thermoacoustic effect. This phenomenon of microwaves was once known as the microwave-auditory, or the microwave-hearing, effect in electromagnetically lossy media.(5) Later, laser technology provided a useful short-pulsed and wavelength-tunable light source for PAT. At some wavelengths of light, the absorption coefficient of blood can be 10 times higher than that of its surrounding tissues, which results in excellent intrinsic contrast for blood-vessel imaging. PAT has been successfully applied to imaging vascular structures and tumor angiogenesis a few mm under the skin.(6-9) However, due to the strong optical scattering effect in biological tissues, which enhances the effective optical absorption, light intensity and, hence, photoacoustic strength, decreases with depth approximately exponentially, with a decay constant of a few $\mathrm{mm}$. Esenaliev et al. studied the imaging sensitivity of PAT on deeply embedded objects using tissue phantoms,(10) and Oraevsky et al. reported a PAT image of a ductal carcinoma at a depth of about $1.1 \mathrm{~cm}$ from the breast surface using a 1064-nm wavelength.(11,12)

\footnotetext{
${ }^{*}$ To whom all correspondence should be addressed: Telephone: 979-847-9040; Fax: 979-845-4450; Electronic mail: LWang@tamu.edu; URL: http://oilab.tamu.edu.
} 
The pulse of electromagnetic waves in the radiofrequency and microwave bands can generate acoustic signals in biological tissues. In the 1980's, several investigators employed microwave-induced thermoacoustics to image biological tissues.(13-18) These early works did not produce significant tomographic images but did prove that microwave-induced thermoacoustic signals could be used for imaging purposes. Later, depth-resolved images in biomedical tissues were computationally reconstructed (19-21) or directly formed by the linear scanning of a focused ultrasound transducer. The latter technique is similar to an ultrasonic B-scan, except that the ultrasound is produced inside the tissue by microwave pulses.(22-24) Kruger et al. reported on thermoacoustic breast cancer imaging in vivo and called it thermoacoustic CT.(25,26)

The generation, propagation, and detection of induced acoustic signals can be described by thermal expansion, wave equations and bandwidth filtering, respectively. ${ }^{27}$ By directing the laser or microwave pulses to a test sample, the absorbed electromagnetic energy in the tissue is transformed into thermal energy, which is then, due to thermo-elastic expansion, converted into mechanical stress. The energy deposition occurs rapidly in a time period that is much less than the thermal relaxation time and the stress relaxation time. The local pressure rise after heating can be derived as $\Delta p=\frac{\beta c^{2}}{C_{p}} E_{a}$, where $\Delta p$ is the pressure rise; $\beta$ is the isobaric volume expansion coefficient; $c$ is the speed of sound; $C_{p}$ is the specific heat; and $E_{a}$ is the absorbed optical energy density. The behavior of photoacoustic or thermoacoustic waves was studied by Diebold and Gusev et al. based on the following non-homogenous wave equation: $:^{28,29}$

$$
\nabla^{2} p(\mathbf{r}, t)-\frac{1}{c^{2}} \frac{\partial^{2} p(\mathbf{r}, t)}{\partial t^{2}}=-\frac{\beta}{C_{p}} \frac{\partial H(\mathbf{r}, t)}{\partial t},
$$

where $p(\mathbf{r}, t)$ is the acoustic pressure at time $t$ and position $\mathbf{r}$ and $H(\mathbf{r}, t)$ is the heat function of the optical energy deposited in the tissues per unit volume per unit time, which can be expressed as

$$
H(\mathbf{r}, t)=A(\mathbf{r}) I(t),
$$

where $A(\mathbf{r})$ describes the optical or microwave energy deposition within the tissues at position $\mathbf{r}$ (structure of tissues); and $I(t)$ describes the shape of the irradiation pulse, which can be further expressed as $I(t)=\delta(t)$ for impulse heating. The purpose of photoacoustic tomography is the reconstruction of the distribution of the optical absorption $A(\mathbf{r})$ in the tissues from a set of measured acoustic signals $p(\mathbf{r}, t)$. For a circular scanning configuration, the exact inverse solution can be derived. In most practical image reconstructions, the algorithm can be simplified to shorten the reconstruction time. If the detection radius $r_{0}$ is much longer than the wavelength that corresponds to the central frequency of the detection device (far-field detection), we can assume $|k| r_{0}>>1$ and simplify the exact inverse solution to the form of ${ }^{30}$

$$
A(\mathbf{r})=-\left.\frac{\beta}{2 \pi c^{4} C_{p}} \iint_{S_{0}} d S_{0}(\cos \Theta) \frac{1}{t} \frac{\partial p\left(\mathbf{r}_{0}, t\right)}{\partial t}\right|_{t=\left|\mathbf{r}_{0}-\mathbf{r}\right| / c}
$$

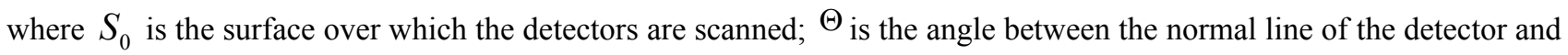
the vector from the detector to the reconstruction point.

\section{EXPERIMENTAL SETUP}

Figure 1 illustrates the experimental setup of optical and electrical contrast (dual contrast) imaging. A laser pulse, which is generated from a Q-switched Nd:YAG laser, or its second harmonic oscillation, at a repetition rate of 10 $\mathrm{Hz}$, provides $\sim 10$-ns near infrared or green laser pulses at a wavelength of 1064 or $532 \mathrm{~nm}$, respectively. The fundamental and second harmonic Q-switched Nd:YAG laser can provide $1 \mathrm{~J}$ or $400 \mathrm{mj}$ output energy, respectively. The laser beam is expanded by a concave lens, homogenized by a ground glass and then directed onto a sample. When the system is switched to TAT, the microwave pulses transmitted from a $3-\mathrm{GHz}$ microwave generator are delivered to the sample through a rectangular waveguide and then an air-filled pyramidal horn antenna with an opening of $120 \mathrm{~mm} x$ $88 \mathrm{~mm}$. The pulse repetition rate is set to around $50 \mathrm{~Hz}$ for the imaging. The pulse width is measured to be $0.5 \mu \mathrm{s}$, and the pulse energy is estimated to be $15 \mathrm{~mJ}$. 
Both PAT and TAT systems have multiple detection channels that can simultaneously receive thermoacoustic signals through multiple ultrasonic transducers. It is difficult to achieve both high imaging resolution (wide bandwidth) and high sensitivity using a single ultrasonic transducer. Hence, ultrasonic transducers with different central frequencies are employed to expand the detection bandwidth. PAT and TAT systems are capable of simultaneously imaging with multiple detection channels, which allows us to employ various ultrasonic transducers and optimize the detection parameters more efficiently. The nanoseconds laser pulse is much shorter than that of a microwave pulse $(0.5$ microseconds), and the photoacoustic signals have a broader spectrum. Therefore, higher frequency transducers can be employed in photoacoustic tomography for better imaging resolution. Generally, ultrasonic transducers, with central frequencies ranging from $2 \mathrm{MHz}$ to $10 \mathrm{MHz}$, are used for PAT; while $1 \mathrm{MHz}$ to $5 \mathrm{MHz}$ ultrasonic transducers are used for TAT. Two $0.6 \mathrm{~cm}$ diameter ultrasonic transducers of $2.25 \mathrm{MHz}(\mathrm{V} 323 / 2.25 \mathrm{MHz}$, Panametrics) and $3.5 \mathrm{MHz}$ (V383/3.5-MHz, Panametrics) are typically employed in both PAT and TAT experiments. The transducers are fastened to a rotational apparatus and immersed in mineral oil in a plastic container. A step motor drives the rotational apparatus and then scans the transducers around the sample along a horizontal circle with the transducers always pointing horizontally to the rotation center during the scanning. The ultrasonic transducers convert the thermoacoustic energy into electronic signals, which are then amplified and filtered electronically. The electronic signals are recorded by a Gage card (CompuScope 14200, Gage Applied Technologies), averaged 20 to 100 times at each scanning stop, and then transferred to a computer for imaging based on back-projection algorithms. The sampling frequency of the oscilloscope is set to 20 or $50 \mathrm{MHz}$. Each scanning takes approximately 10 to 30 minutes.

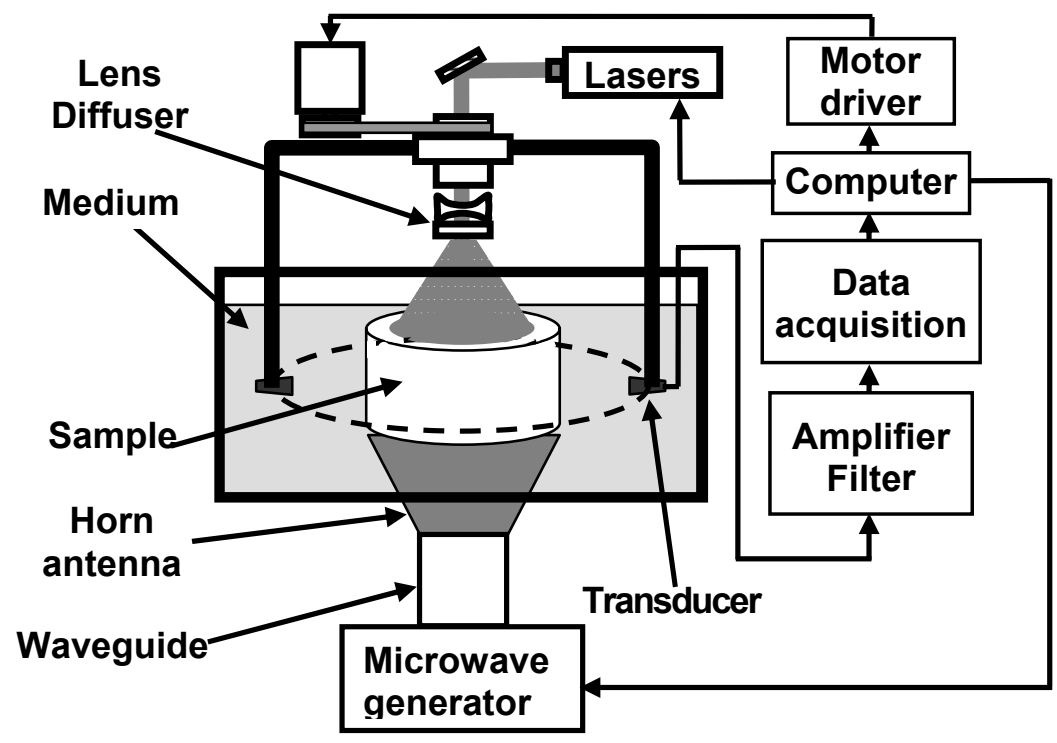

Figure 1. Experimental setup of dual contrast imaging.

\section{RESULTS AND DISCUSSION}

Figure 2 shows a test sample for dual contrast test. Three objects made of lard, blue gel, and white gel, respectively, are buried in a piece of porcine fat of around $10 \mathrm{~cm}$ in its diameter. Other porcine fat blocks will covered the objects and make them 2-3 cm deep from the surface. The blue gel possesses both optical and microwave absorption because of its blue color and high water content, respectively; the lard object has little difference from the porcine fat tissue around it as lard was extracted from porcine fat. The white gel looks white as the porcine fat but quite different in electrical properties. 


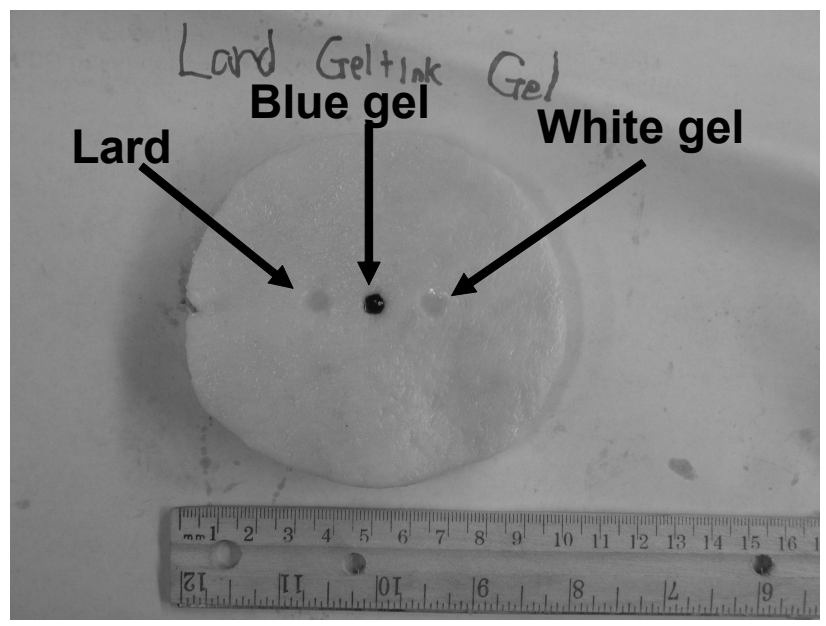

Figure 2. Photograph of test sample I (Lard, blue gel and white gel in porcine fat).

The photoacoustic and thermoacoustic images of the above sample are compared in Fig. 3. The blue gel is clearly imaged by both photoacoustic and thermoacoustic tomography as expected. In the photoacoustic image, the lard and white gel objects can also be discerned although their image intensities are much lower than that of blue gel object. The respective appearances of the lard and the white gel in the photograph demonstrate their optical contrasts. In addition to optical absorption, other physical properties, such as the thermo expansion coefficient and the acoustic speed and heat capacity, may also have contributed to the image contrast. This photoacoustic image reveals that photoacoustic tomography is very sensitive to optical contrast. The lard object is not presented in a thermoacoustic image, but both gel objects are exhibited, and they effectively demonstrate the sensitivity of thermoacoustic imaging to water content.
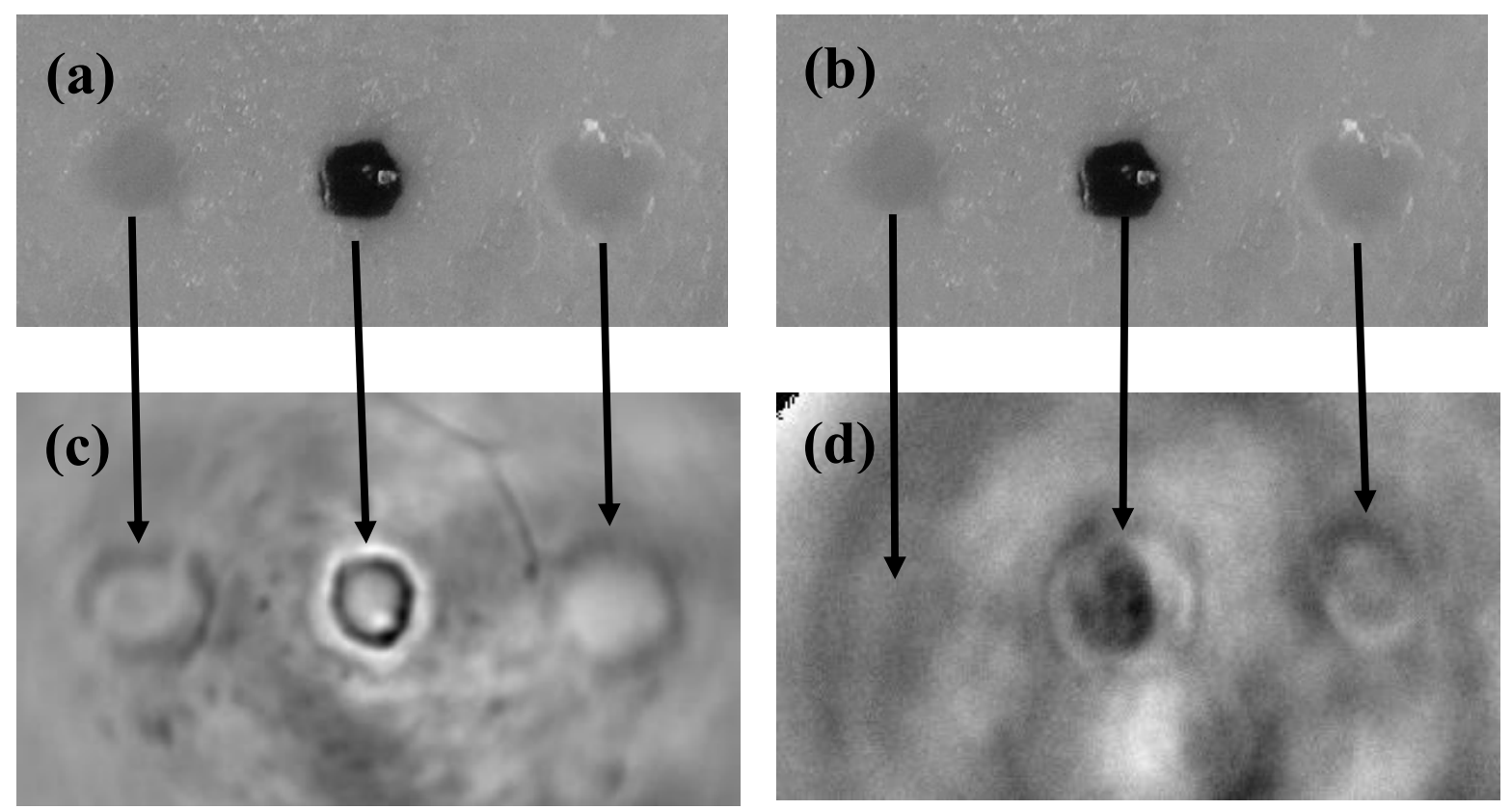

Figure 3. Photoacoustic and thermoacoustic images comparison. (a) and (b) Zoom-in photograph of test sample. Sample's image by (c) photoacoustic, and (d) thermoacoustic tomography. 
We made an all tissue sample by burring porcine tendon and muscle objects in a porcine fat [Fig. 4 (a)]. The muscle object has both optical and microwave absorption because of its high blood red cell and water content. The tendon was taken from pig foot and had two layers. The outer layer of the tendon looks like a white tube, which is consisted of connective tissues of high water content; while the inner part of the tendon is a yellowish cylinder that presents different color from that of the outer white tube. The 1D photoacoustic and thermoacoustic image across the buried tendon and muscle objects are shown in Fig. 4(b). The 2D images by photoacoustic and thermoacoustic tomography are shown in Fig. 4(c) and 4(d), respectively. The muscle object is clearly imaged by both thermoacoustic and photoacoustic tomography. The tendon object exhibits slightly different structure in photoacoustic and thermoacoustic images. It appeared that the photoacoustic tomography images the inner cylinder of the tendon and thermoacoustic tomography presents the outer white tube of the tendon. Using only one image contrast, the whole tendon structure can not be recognized; however, the tendon constitution can be identified by comparing its thermoacoustic and photoacoustic images.

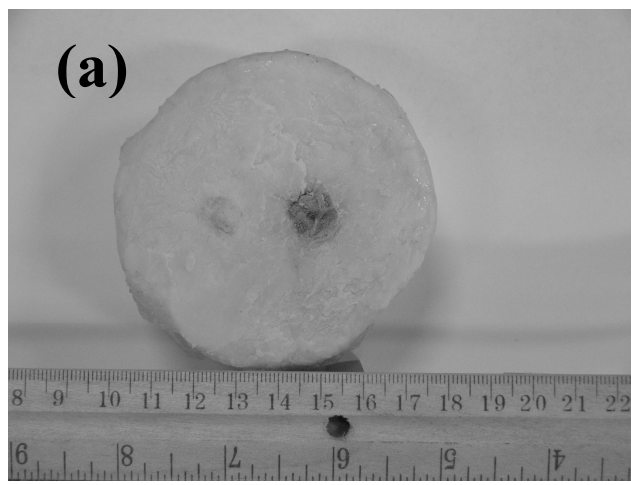

\section{(b)}
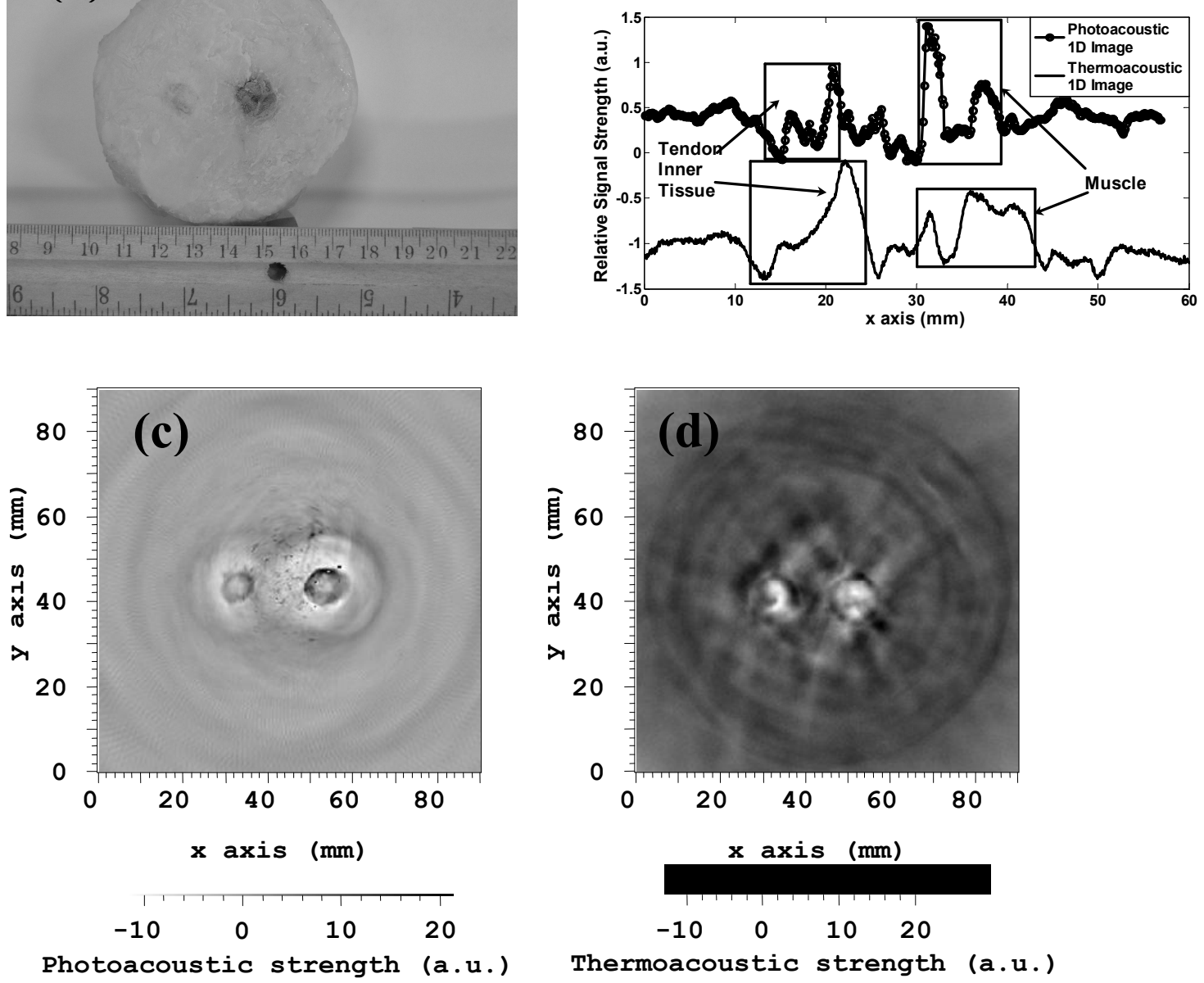

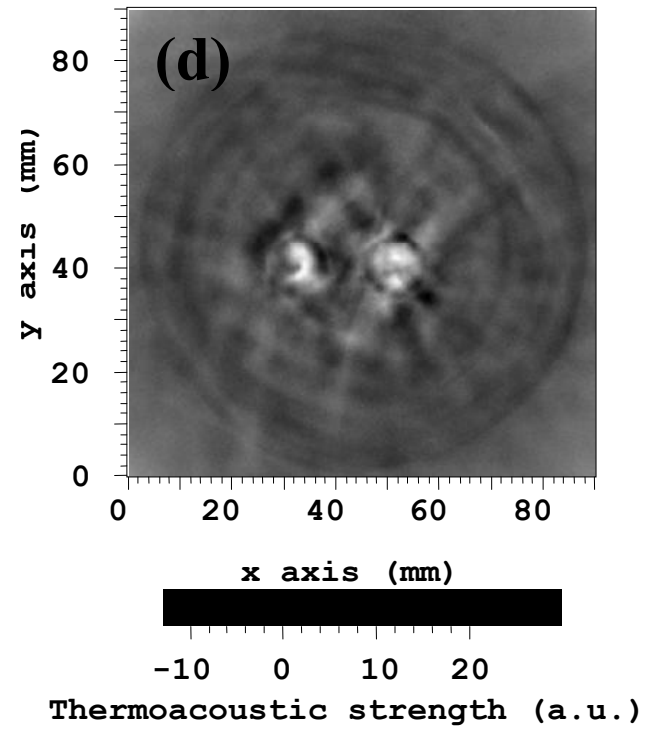

Figure 4. Tissue test sample and its photoacoustic and thermoacoustic images comparison. (a) Photograph of the test sample of tendon and muscle objects in porcine fat. (b) One-dimensional photoacoustic and thermoacoustic image across tendon and muscle objects. Two-dimensional images by (c) photoacoustic and (d) thermoacoustic tomography. 


\section{CONCLUSION}

Our experiments demonstrate that combining photoacoustic and thermoacoustic tomography can disclose both the optical and electrical properties of tissues simultaneously. The dual imaging contrasts are complementary for some biological tissue components and may reveal tissues properties more comprehensively. The imaging depth reaches 2- 3 $\mathrm{cm}$ from the illuminated surface in these experiments but can be extended to $5 \mathrm{~cm}$ depending on the signal-to-noise ratio.

\section{ACKNOWLEDGEMENT}

We thank Meng-Lin Li, Jung-Taek Oh and Xueyi Xie, Ryan Lawless, Xing Jin and Manojit Pramarik for assistance on photoacoustic and thermoacoustic tomography, respectively.

\section{REFERENCES}

1 S. Chaudhary, R. Mishra, A. Swarup, and J. Thomas. Dielectric Properties of Normal \& Malignant Human Breast Tissues at Radiowave and Microwave Frequencies. Indian J. Biochem. Biophys. 21, 76-79 (1984).

2 W. Joines, R. Jirtle, M. Rafal, and D. Schaeffer. Microwave Power Absorption Differences between Normal and Malignant tissue. Radiation Oncology-Biology-Physics. 6, pp. 681-687 (1980).

3 W. Joines, Y. Zhang, C. Li, and R. Jirtle. The Measured Electrical Properties of Normal and Malignant Human Tissues from 50-900 MHz. Medical Physics. 21, 547-550 (1994).

4 W. F. Cheong, S. A. Prahl, A. J. Welch. A Review of the Optical Properties of Biological Tissues. IEEE J. Quantum Elect. 26, 2166-2185 (1990).

5 J. C. Lin. On Microwave-Induced Hearing Sensation. IEEE Trans. Microwave Theory Tech. 25, 605-613 (1977).

6. R. G. M. Kolkman, E. Hondebrink, W. Steenbergen, F. F. M. de Mul. In Vivo Photoacoustic Imaging of Blood Vessels Using an Extreme-Narrow Aperture Sensor. IEEE J. Sel. Top. Quantum Electron. 9, 343-346, (2003).

7. X. Wang, Y. Pang, G. Ku, X. Xie, G. Stoica, and L.-H. Wang. Non-Invasive Laser-Induced Photoacoustic Tomography for Structural and Functional Imaging of the Brain In Vivo. Nat. Biotechnol. 21, 803-806 (2003).

8. G. Ku, X. Wang, X. Xie, G. Stoica, L.-H. V. Wang. Multiple-Bandwidth Photoacoustic Tomography. Phys. Med. Biol. 49, 1329-1338 (2004).

9 G. Ku, X. Wang, X. Xie, G. Stoica, L. -H. Wang. Imaging of Tumor Angiogenesis in Rat Brains In Vivo by Photoacoustic Tomography. Applied Optics, 44, 770-775 (2005).

10. R. O. Esenaliev, A. A. Karabutov and A. A. Oraevsky. Sensitivity of Laser Opto-Acoustic Imaging in Detection of Small Deeply Embedded Tumors. IEEE J. Sel. Top. Quant. 5, 981-988 (1999).

11. A. A. Karabutov, E. V. Savateeva, A. A. Oraevsky. Optoacoustic Tomography: New Modality of Laser Diagnostic Systems LASER PHYSICS. 13, 711-723 (2003).

12. A. A. Oraevsky, E. V. Savateeva, S. V. Solomatin, A. A. Karabutov, V. G. Andreev, Z. Gatalica, T. Khamapirad, and P. M. Henrichs. Optoacoustic Imaging of Blood for Visualization and Diagnostics of Breast Cancer. in Biomedical Optoacoustics III, A. A. Oraevsky, ed. 4618, 81-94 (2002).

13 T. Bowen, L. Nasoni, A. E. Pifer, and G. H. Sembrosk, in Proc. IEEE Ultrasonics Symposium 2, 823-827 (1981).

14 J. P. Powers, ed., Acoustic Imaging (Plenum Publishing, New York, 1982).

15 R. G. Olsen and J. C. Lin. Acoustic Imaging of a Model of a Human Hand Using Pulsed Microwave Irradiation. Bioelectromagnetics. 4, 397-400 (1983).

16 J. C. Lin and K. H. Chan. Microwave Thermoelastic Tissue Imaging System Design. IEEE Trans. Microwave Theory Tech. 32, 854-860 (1984). 
17 R. L. Nasoni, G. A. Evanoff Jr., P. G. Halverson, and T. Bowen. Thermoacoustic Emission by Deeply Penetrating Microwave Radiation, in Proc. IEEE Ultrasonics Symposium 5, 633-637 (1984).

18 K. H. Chan and J. C. Lin. Microwave-Induced Thermoacoustic Tissue Imaging. in Proc. Engineering in Medicine and Biology Society 10th Annual International Conference. 445-446 (1988).

19 R. A. Kruger, P-Y. Liu and Y. Fang. Photoacoustic Ultrasound (PAUS) - Reconstruction Tomography. Med. Phys. 22, 1605-1609 (1995).

20 R. A. Kruger, K. K. Kopecky, A. M. Aisen, D. R. Reinecke, G. A. Kruger, and W. L. Kiser Jr. Thermoacoustic CT with Radio Waves: A Medical Imaging Paradigm. Radiology. 211, 275-278 (1999).

21 R. A. Kruger, D. R. Reinecke, and G. A. Kruger. Thermoacoustic Computed Tomography-Technical Considerations. Med. Phys. 26, 1832-1837 (1999).

22 L.-H. Wang, X. Zhao, H. Sun, and G. Ku. Microwave-Induced Acoustic Imaging of Biological Tissues. Rev. Sci. Instrum. 70, 3744-3748 (1999).

23. G. Ku and L.-H. Wang. Scanning Thermoacoustic Tomography in Biological Tissue. Med. Phys. 27, 1195$1202(2000)$.

24 G. Ku and L.-H. Wang, "Scanning Microwave-Induced Thermoacoustic Tomography: Signal, Resolution, and Contrast. Med. Phys. 28, 4-10 (2001).

25 R. A. Kruger and W. L. Kiser Jr. Thermoacoustic CT of the Breast: Pilot Study Observations. Proc. SPIE, 4256:1-5. (2001).

26 R. A. Kruger, K. Stantz, and W. L. Kiser Jr. Thermoacoustic CT of the Breast. Proc. SPIE, 4682:521-525. (2002).

27. G. Ku, L.-H. Wang, "Scanning thermoacoustic tomography in biological tissue," Med. Phys., vol. 27, no. 5 pp. 1195-1202, May 2000.

28. G. J. Diebold, T. Sun, and M. I. Khan, "Photoacoustic waveforms generated by fluid bodies," in Photoacoustic and Photothermal Phenomena III, edited by D. Bicanic, Springer-Verlag, pp. 263-269, Heidelberg, Berlin, 1992.

29. V. E. Gusev and A. A. Karabutov, Laser Optoacoustics. American Institute of Physics, New York: 1993.

30. M. H. Xu, Yuan Xu and L.-H. V. Wang, "Time-domain reconstruction algorithms and numerical simulations for thermoacoustic tomography in various geometries," IEEE Trans. on Biomed. Eng., Vol. 50, pp.1086-1099, Sep. 2003. 